\title{
Early Mobilization in the ICU: An Update
}

\author{
Mark Sigler MD
}

Over the past 10 to 15 years, a culture shift in ICU care has occurred at several leading institutions across the United States. The traditional model of deep anesthesia with prolonged bedrest in mechanically ventilated patients has been replaced with a model of minimal sedation with early mobilization. ${ }^{1}$ In 2000, Kress reported a landmark study addressing sedation minimization by daily interruption of sedative infusions in mechanically ventilated patients. This study evaluated 128 adult patients who were mechanically ventilated and received continuous sedative infusion; the intervention group (who received daily sedation holidays) had a decrease in duration of mechanical ventilation of 2.4 days per patient in comparison to the control group (interruptions in sedation at the discretion of the attending physician) and a decrease in length of ICU stay by 3.5 days per patient. ${ }^{2}$ This contributed to an ICU culture change over the next decade, in which more intensive efforts to minimize sedation were made. In a follow-up study published in JAMA in 2012, daily sedation holidays were re-examined by comparing daily sedation interruption combined with a protocol based sedation strategy (designed to reduce overall sedation) to a protocol based sedation strategy alone. In this study, neither the duration of mechanical ventilation nor length of ICU stay was reduced by the addition of daily sedation interruption. The authors concluded that this likely reflected positively on the change in sedation strategy over 10 years following the initial study by Kress. Rather than the usual care of deep sedation in mechanically ventilated patients that resulted in a clinical benefit from daily sedation interruption, the more current model of global sedation minimization now negated the previous beneficial effects of daily sedation interruption. ${ }^{3}$

Corresponding author: Mark Sigler, MD Contact Information: Mark.sigler@ttuhsc.edu DOI: 10.12746/swrccc2013.0207.085
In addition to minimizing sedation, a strategy of analgesia only versus analgesia plus sedation in mechanically ventilated patients has also been examined. In 2010, The Lancet published a randomized controlled trial that compared mechanically ventilated patients who were given analgesia with morphine and sedation with propofol or midazolam to mechanically ventilated patients who received analgesia with morphine boluses and no scheduled sedation (only rescue propofol was permitted). Patients managed with analgesia only had an average decrease in ventilator time of 4.2 days and a decreased average length of ICU stay of 9.7 days. ${ }^{4}$ The decrease in sedation resulting from these studies has allowed more aggressive interventions to be performed in the arena of early mobilization and physical and occupational therapy. The remainder of this article will focus on the data evaluating early mobilization and early implementation of physical and occupational therapy in the ICU.

In 2003, investigators at LDS Hospital in Salt Lake City conducted a study in which patients requiring mechanical ventilation were assessed for early activity (sitting on the bed, sitting in the chair, and ambulation). Over the course of seven months, over 1400 activities were performed with more than 100 patients; less than $1 \%$ of these activities had an activity-related adverse event (including zero extubations). This sentinel study concluded that early activity was both feasible and safe in patients on mechanical ventilators. ${ }^{5}$

Research at Wake Forest University addressed the specific timing of mobility therapy. Within 48 hours of initiation of mechanical ventilation, a mobility protocol was begun; this study showed a reduction of 1.4 days in ICU length of stay for patients in the intervention arm and a three day decrease in length of hospital stay. In addition, there were no adverse events during therapy sessions. This trial concluded that early mobilization was both safe and effective in the early stages of mechanical ventilation. ${ }^{6}$ Similar re- 
sults were found by Schweickert in a study published by the Lancet in 2009 , which showed a statistically significant decrease in ventilator-free days in patients who received early exercise and mobilization during daily interruption of sedation. ${ }^{7}$

Following these studies, the next issue to be addressed was the feasibility and safety of early mobilization in patients who have traditionally been considered unstable. Pohlman and Kress evaluated this in 2010 by starting physical and occupational therapy in mechanically ventilated patients approximately 1.5 days after initiation of mechanical ventilation. During $35 \%$ of the sessions, the $\mathrm{FiO}_{2}$ was $>0.60$ and in $31 \%$, one or more vasoactive drugs were being infused at the time of the mobilization session. Only $4 \%$ of all sessions were stopped prematurely (usually due to patient-ventilator asynchrony or agitation). Thus, this study concluded that even in patients with high acuity of illness early physical and occupational therapy was feasible. ${ }^{8}$

Despite the evidence supporting the minimization of sedation and institution of early mobilization in the critical care environment, it can be challenging to put these concepts into clinical practice. Barriers include education of both physicians and nurses regarding appropriate sedation and analgesia regimens, failure to attempt mobilization of acutely ill patients, and failure to involve physical and occupational therapy services early in the hospital course.

In April 2014, University Medical Center in Lubbock, Texas, initiated "Early Mobilization in the Medical Intensive Care Unit", a quality improvement project aimed at improving the mobilization of patients admitted to the MICU. Similar projects had previously been attempted at this hospital with minimal success. In an effort to create lasting change, a five-pronged approach was instituted, and to ensure multi-disciplinary involvement, representatives from physicians, nurses, physical and occupation therapy, and respiratory therapy were consulted in the development of the project.

Five areas for improvement were targeted at the University Medical Center MICU:
1. Review of the sedation and analgesia regimen on a daily basis. This would ensure that all patients had adequate analgesia prior to initiating or increasing sedation.

2. Implementing daily sedation interruptions. Because the culture of the MICU emphasized higher amounts of sedation, it was felt that the patients would benefit from daily sedation interruption.

3. Implementation of a "Progressive Mobility Protocol" on every patient admitted to the MICU. The "Progressive Mobility Protocol" is a nursing-driven, eight-step order set that progressively advances the activity of each patient. This eight-step protocol is placed on the door of each patient's room, with the specific step that each patient has achieved circled with an erasable marker; every eight hours, further advancement on this protocol is attempted.

4. Early consultation of physical and occupational therapy. Once a patient has reached step three on the progressive mobility protocol (achieving a chair position in bed with feet on the floor), physical and occupational therapy consults are requested to assist with further mobilization.

5. Passive range of motion exercises for every patient. The Physical Medicine and Rehabilitation department at University Medical Center developed a 10-exercise program of passive range of motion exercises to be performed three times per day for every patient, regardless of which stage they have reached on the "Progressive Mobility Protocol".

During the month prior to the initiation of the quality improvement project, members of the team met with all MICU nursing staff, physical and occupational therapy staff, respiratory therapy staff, and the interns and residents who would be providing care in the MICU. The five pillars of the early mobilization project were addressed with time allowed for questions and answers. On April 1, 2014, the "Early Mobilization in the Medical Intensive Care Unit" quality improvement project was begun. On a daily basis, the nursing directors of the MICU have assessed whether the five pillars of the project are being addressed 
and implemented on each patient. While the project is scheduled to be performed for six months, early success has been noted. Less than two weeks after project initiation, the University Medical Center MICU nursing staff, with the assistance of physical and occupational therapy and respiratory therapy, achieved its initial successful ambulation of a mechanically ventilated patient.

At the conclusion of the quality improvement project, a data analysis will be performed to assess impact on both length of ICU stay and length of time on mechanical ventilation. Complications and adverse events will also be reviewed.

Acknowledgements: Special thanks to the multi-disciplinary team that was instrumental in developing this project: Raed Alalawi MD, Connie Garcia RN, Anna Geesling OTR, Alisha Holleman RN, Sarah Johnson RN, David Krause PT, John Lowe PT, Chad Montandon RN, Kenneth Nugent MD, Madelyn Sparks RN, Heath Stark RTA, Irene Torres RRT, Kristi Valdez RN and Hazel Violan RN.

Author Affiliation: Mark Sigler is a pulmonary fellow in the Department of Internal Medicine at TTUHSC.

Received: 4/12/2014

Accepted: 6/30/2014

Reviewers: Raed Alalawi MD

Published electronically: 7/15/2014

Conflict of Interest Disclosures: None critically ill patients cared for with a sedation protocol: a randomized controlled trial. JAMA 2012; 308(19):1985-1992.

4. Strom, Thomas; Martinussen, Torben; and Toft, Palle. A protocol of no sedation for critically ill patients receiving mechanical ventilation: a randomized trial. The Lancet 2010 Feb 6; 375(9713):475-80.

5. Bailey, Polley; Thomsen, George E, and Spuhler, Vicki $\mathrm{J}$ et al. Early activity is feasible and safe in respiratory failure patients. Crit Care Med 2007; 35(1):139-145.

6. Morris, Peter E; Goad, Amanda; and Thompson, Clifton, et al. Early intensive care unit mobility therapy in the treatment of acute respiratory failure. Crit Care Med 2008; 36(8): 2238-2244.

7. Schweickert, William D; Pohlman, Mark C; and Pohlman, Anne S. Early physical and occupational therapy in mechanically ventilated, critically ill patients: a randomized controlled trial. The Lancet 2009; 373:1874-82.

8. Pohlman, Mark C; Schweickert, William D; and Kress, John P et al. Feasibility of physical and occupational therapy beginning from initiation of mechanical ventilation. Crit Care Med 2010; 38(11):2089-2094.

\section{REFERENCES}

1. Schweickert, William D and Kress, John P. Implementing early mobilization interventions in mechanically ventilated patients in the ICU. CHEST 2011; 140(6):16121617.

2. Kress, John P; Pohlman, Anne S; and O'Connor, Michael $\mathrm{F}$ et al. Daily interruptions of sedative infusions in critically ill patients undergoing mechanical ventilation. New Engl J Med 2000; 342:1471-7.

3. Mehta, Sangeeta; Burry, Lisa; and Cook, Deborah et al. Daily sedation interruption in mechanically ventilated 